\title{
PARAMETROS GENÈTICOS DE CARACTERİSTICAS PRODUCTIVAS Y REPRODUCTIVAS PARA GANADO TIPO CARNE EN COLOMBIA.
}

\section{GENETIC PARAMETERS FOR PRODUCTIVE AND REPRODUCTIVE CHARACTERISTICS FOR BEEF CATTLE IN COLOMBIA.}

\author{
MONTES-V, DONICER ${ }^{1 * E s p, ~ B A R R A G A ́ N-H, ~ W I L S O N ² ~ Z o o t e c, ~ V E R G A R A-G, ~}$ \\ OSCAR ${ }^{3} \mathrm{Dr}$.
}

1Universidad de Sucre, Facultad de Ciencias Agropecuaria, Departamento de Zootecnia, Sincelejo, Colombia. 2 Ejercicio Particular. 3 Universidad de Córdoba,

Facultad de Medicina Veterinaria y Zootecnia. Departamento de Ciencias

Pecuarias. Montería, Colombia.

Grupo Reproducción y Mejoramiento Genético Animal

*Correspondencia:donicermontes@hotmail.com

\begin{abstract}
Resumen
Con el propósito de presentar la panorámica de las investigaciones realizadas en mejoramiento genético animal para ganado de carne en Colombia, fueron revisados 27 artículos, publicados en revistas reconocidas en el ámbito nacional y en lo posible disponibles en la red., Las publicaciones fueron agrupadas en dos categorías: características productivas y reproductivas. Para las características productivas se referenciaron trabajos publicados para las variables peso al nacimiento (PN) con valores medios de $h_{d}{ }^{2}, h_{m}{ }^{2}$ y $h_{t}{ }^{2}$ de $0.23,0.15$ y 0.22 respectivamente, de igual manera para el peso al destete (PD) con valores de $0.21,0.12$ y 0.21 , respectivamente; así mismo se reportan, aunque en menor cantidad, publicaciones para los pesos a los 12, 16 y 18 meses. Para las características reproductivas, solo se hallaron dos trabajos publicados para la edad al primer parto (EPP), ambos con una $h_{d}{ }^{2}$ de $0.16 \pm 0.086$, y para el intervalo entre partos (IEP) valores que oscilan entre $0.0 \pm 0.06$ y $0.15 \pm 0.07$. De igual manera fueron reportados estimativas de repetibilidad y correlaciones genéticas, fenotípicas y ambientales para las características productivas.
\end{abstract}

Palabras clave: Heredabilidad, correlaciones, características productivas y reproductivas, bovinos. 


\begin{abstract}
In order to present the overview of research in animal genetic improvement of beef cattle in Colombia, 27 published articles were reviewed in recognized national journals available on the web; publications were grouped into two categories: productive and reproductive characteristics. For the production characteristics were referenced published papers for the variables birth weight (BW) with mean values of $\mathrm{h}_{\mathrm{d}}{ }^{2}, \mathrm{~h}_{\mathrm{m}}{ }^{2} \mathrm{y} \mathrm{h}_{\mathrm{t}}{ }^{2}$ of $0.23,0.15$ and 0.22 respectively, similarly the weight at weaning (PD) with values of $0.21,0.12$ and 0.21 was registered, Likewise some published papers reported heritability although in smaller quantities for the weights at 12, 16 and 18 months. For reproductive features only two published works were found for age at first birth (AFB), both with a hd2 of $0.16 \pm 0.086$, and for calving interval (CI) values ranging from $0.0 \pm 0.06$ and $0.15 \pm 0.07$. In the same light, estimates of repeatability genetic correlations and phenotypic and environmental production characteristic were reported,
\end{abstract}

Key words: Heritability, correlations, productive and reproductive characteristics, Cattle

\title{
Introducción
}

La actividad ganadera en Colombia participa con el 3.6\% del PIB Colombiano, valor que la posiciona en el decimo primer lugar, superando sectores agrícolas y de servicios de mucha importancia en el país (FEDEGAN, 2006).

Colombia, actualmente cuenta con 26.887 .824 bovinos, de los cuales el $67 \%$ corresponde a bovinos dedicados a la ganadería de carne y/o con aptitud para esta explotación. El inventario presentó un crecimiento del $1.4 \%$ con relación a los 2 años anteriores, (MADR, 2008). Este crecimiento evidencia un avance desde el punto de vista productivo.

El mejoramiento genético es uno de los pilares fundamentales de las explotaciones ganaderas, este tiene como objetivo alterar la frecuencia deseable de genes en una población (OSSA et al., 2008). Los parámetros genéticos son de extrema importancia en este proceso, ya que constituyen las herramientas utilizadas para la obtención de las respuestas directas y correlacionadas de la selección, elaboración de índices de selección y predicción de los valores genéticos de los animales (DE LIRA et al., 2008), generando así información que nos permite identificar aquellos animales sobresalientes o con merito genético, capaces de producir eficientemente y de trasmitir su potencial a su descendencia. 
La heredabilidad se presenta como uno de los parámetros genéticos más importantes en el mejoramiento genético animal. FALCONER (1980),la define como el coeficiente de la varianza genética aditiva sobre la varianza fenotípica, y nos define la capacidad de trasmisión de determinado carácter a su descendencia. Las correlaciones genéticas se presentan como otra herramienta fundamental para el mejoramiento genético, permite predecir cómo se comportará determinada característica con relación al desempeño de otra, afectándola de forma positiva o negativa (FALCONER y MACKAY, 1996).

El objetivo de esta revisión es presentar el panorama de la investigación en mejoramiento genético para ganado de carne en Colombia a través de información publicada y disponible, con la finalidad de concientizar a la comunidad científica de la importancia del mejoramiento genético como uno de los pilares fundamentales para aumentar los parámetros productivos y reproductivos en la ganadería de carne colombiana.

\section{Descripción de la información}

Fueron utilizados 27 artículos publicados entre los años 1998 y 2009, en los que se evaluaron parámetros genéticos de características productivas y reproductivas para ganado tipo carne en Colombia., La información, referente al tema, fue seleccionada de artículos de revistas de reconocimiento nacional e internacionales de circulación impresa o electrónica

La información de los parámetros genéticos hallados se agruparon en dos categorías: productivas y reproductivas., La primera abarco características como peso al nacimiento (PN), peso al destete (PD), el cual generalmente fue ajustado a los 205 y 270 días; peso a los 12 (P12), 16 (P16) y 18 meses (P18); y las reproductivas solo intervalo entre partos (IEP) y edad al primer parto (EPP).

\section{Características productivas}

Peso al Nacimiento. El peso al nacer (PN) se presenta como el primer registro que se obtiene de un animal para comenzar su vida productiva, está asociado a pesos posteriores, y es de gran importancia como base para la selección, con el objeto de evitar partos distócicos y alcanzar pesos al sacrificio en un periodo más corto (OSSA et al., 2005a).

Para esta característica productiva, 12 de los 27 artículos revisados estimaron parámetros de heredabilidades directas $\left(h_{d}{ }^{2}\right)$, maternas $\left(h_{m}{ }^{2}\right)$ y totales $\left(h_{t}{ }^{2}\right)$. Las $h_{d}{ }^{2}$ variaron de $0.08 \pm 0.03$ (OSSA et al., 2002) en la raza Costeño Con Cuernos (CCC) 
hasta $0.39 \pm 0.05$ (GALLEGO et al., 2008) para ganado Blanco Oreginegro (BON). Para la $\mathrm{h}_{\mathrm{m}}{ }^{2}$, los estimados oscilaron entre $0.012 \pm 0.003$ para la raza CCC (MARTÍNEZ et al., 2006a) y 0.36 para animales tipo Cebú no definidos (ELZO et al., 2006) y estimados de parámetros genéticos de PN para $h_{t}^{2}$ solo fueron presentados por MARTíNEZ et al. $\left(2006^{\mathrm{a}}, 2006 \mathrm{~b}\right)$ para animales CCC y Romosinuano, reportando valores de 0.17 y 0.28 , respectivamente.

Los parámetros hallados para $\mathrm{PN}$ se pueden clasificar como bajos, evidenciando la fuerte influencia ambiental sobre esta característica, no obstante, la gran mayoría de estos estudios se presenta sobre ganado criollo bajo esquemas de conservación genética.

Las medias de los estimados de $h_{d}{ }^{2}, h_{m}{ }^{2}$ y $h_{t}{ }^{2}$ fueron $0.23,0.15$ y 0.22 , respectivamente., El total de registros se presenta en la tabla 1.

Peso al Destete. El peso al destete (PD) que refleja en gran medida la capacidad materna de la vaca y su habilidad para criar terneros (DE LIRA et al., 2008), se presenta como importante punto de partida para la selección animal, ya que el ternero se desteta cerca del octavo mes con, aproximadamente, el 42 al $50 \%$ de su peso adulto (OSSA et al ., 2005b; CAÑAS et al., 2008).

El $51.8 \%$ de los artículos revisados presentaron estimados de heredabilida para esta característica, presentando mayor influencia los parámetros de $h_{d}{ }^{2}$, los cuales oscilaron entre 0.10 (QUINTERO et al., 2007) para animales de la raza Brahman y $0.63 \pm 0.36$ (CAÑAS et al., 2008) para la raza BON.

La característica PD es una medida muy compleja, ya que en ella influyen factores genéticos aditivos del padre y de la madre, factores genéticos aditivos maternos, expresados como un valor fenotípico de la madre (capacidad lechera) y medido como un valor de peso fenotípico expresado en el hijo, efecto del ambiente permanente de la madre y efecto ambiental directo (QUINTERO et al., 2007; CAÑAS et al., 2008), motivo por el cual ,su estimación debe ser muy precisa y abarcar en la totalidad todas las influencias.

QUINTERO et al. (2007) presentaron, en una evaluación de 5 modelos que incluían o no el efecto materno, la posibilidad de sobrestimar la heredabilidad para el PD, cuando no se tienen en cuenta los efectos maternos que influencian esta característica., Los valores para este parámetro oscilaron entre $0.10 \pm 0.03$ y $0.29 \pm 0.038$ para la estimación de la $h_{d}{ }^{2}$ y entre $0.09 \pm 0.048$ hasta $0.17 \pm 0.033$ para la $\mathrm{h}_{\mathrm{m}}{ }^{2}$. Sin embargo en otras evaluaciones, como las reportadas por OSSA et al., (2005), DODENHOFF et al. (1999a) y DODENHOFF et al. (1999b), se afirman que cuando las estimativas de heredabilidad para el efecto materno son menores que 
las estimadas para el efecto directo, el efecto materno no necesariamente debe ser incluido en el modelo, para estimar los parámetros genéticos con mayor exactitud. En la tabla 1 se presentan la totalidad de estudios que estimaron heredabilidad para PD.

Tabla 1. Parámetros de $h_{d}{ }^{2}, h_{m}{ }^{2}$ y $h_{t}{ }^{2}$ estimados para características productivas en ganado tipo carne en Colombia.

\begin{tabular}{|c|c|c|c|c|c|c|c|}
\hline \multirow{2}{*}{ AUTOR } & \multirow{2}{*}{ REVISTA } & \multicolumn{3}{|c|}{ PN } & \multicolumn{3}{|c|}{ PD } \\
\hline & & $h_{d}{ }^{2}$ & $\mathrm{~h}_{\mathrm{m}}{ }^{2}$ & $\mathbf{h}_{\mathrm{t}}^{2}$ & $h_{d}{ }^{2}$ & $\mathrm{~h}_{\mathrm{m}}{ }^{2}$ & $h_{t}^{2}$ \\
\hline ARBOLEDA et al. (2007) & LRRD & & & & 0,08 & 0,08 & \\
\hline CAÑAS et al. (2008) & MVZ & & & & $0,63 \pm 0,36$ & $0,22 \pm 0,19$ & \\
\hline CASTAÑO et al. (2003) & COL CIEN PEC & $0,1 \pm 0,009$ & & & & & \\
\hline ELZO et al.( 1998) & JOUR ANI SCI & 0,16 & 0,18 & & 0,09 & 0,09 & \\
\hline ELZO et al .(1999) & JOUR ANI SCI & 0,24 & 0,14 & & 0,1 & 0,13 & \\
\hline ELZO et al .(2003) & COL CIEN PEC & 0,26 & 0,29 & & 0,18 & 0,11 & \\
\hline ELZO et al. (2003) & COL CIEN PEC & 0,3 & 0,36 & & 0,08 & 0,1 & \\
\hline GALLEGO et al. (2006) & CORPOICA & $0,39 \pm 0,05$ & & & $0,18 \pm 0,05$ & & \\
\hline MANRIQUE (2003) & COL CIEN PEC & 0,48 & & & 0,45 & & \\
\hline MARTINEZ et al. (2006) & CORPOICA & $0,25 \pm 0,0001$ & $0,06 \pm 0,003$ & 0,28 & $0,34 \pm 0,06$ & $0,19 \pm 0,05$ & 0,43 \\
\hline MARTINEZ et al. (2006) & CORPOICA & $0,17 \pm 0,001$ & $0,012 \pm 0,003$ & 0,17 & $0,21 \pm 0,007$ & $0,05 \pm 0,003$ & 0,23 \\
\hline MONTES et al .(2008) & MVZ & 0,16 & & & 0,37 & & \\
\hline OSSA et al .(2007ạ) & CORPOICA & $0,17 \pm 0,06$ & $0,087 \pm 0,03$ & & $0,14 \pm 0,05$ & $0,12 \pm 0,03$ & \\
\hline OSSA et al. (2002) & MVZ & $0,08 \pm 0,03$ & & & $0,26 \pm 0,82$ & & \\
\hline OSSA et al. (2005무) & MVZ & $0,17 \pm 0,06$ & $0,087 \pm 0,03$ & & & & \\
\hline OSSA et al .(2005b) & MVZ & & & & $0,14 \pm 0,05$ & $0,12 \pm 0,03$ & \\
\hline QUINTERO et al.(2007) & COL CIEN PEC & & & & $0,10-0,29$ & $0,09-0,17$ & \\
\hline VERGARA et la. (2009) & JOUR ANI SCI & & & & $0,23 \pm 0,047$ & $0,15 \pm 0,041$ & 0,19 \\
\hline
\end{tabular}




\section{Peso a los 12,16 y 18 meses}

El peso a los 12 meses (P12) se presenta como un indicador de la habilidad del individuo para ganar peso después del destete, asumiendo que los efectos que influyen sobre esta condición son influenciados por efectos genéticos aditivos (padre y madre) y no aditivos (ambiente) (DE LIRA et al., 2008).

El P12 es una característica poco estudiada, solamente un estudio presento estimados de heredabilidad directa, con un valor de 0.4 para la raza Brahman (MANRIQUE, 2003). Esta estimativa puede ser considerada confiable dada la cantidad de registros que fueron evaluados (10.531).

Tres estudios presentaron datos relacionados con la heredabilidad del peso a los 16 meses. MARTINEZ et al. (2006b) y OSSA et al. (2007) para la raza Romosinuano(Romo) y OSSA et al . (2002) para la raza CCC, con heredabilidades directas de $0.33 \pm 0.66,0.13 \pm 0.05$ y $0.22 \pm 0.08$ para las razas Romo y CCC, respectivamente. Los estimados de $\mathrm{h}_{\mathrm{m}}{ }^{2}$ fueron muy bajos $(0.01 \pm 0.04$; MARTINEZ et al., 2006b), lo que evidencia el pobre efecto materno sobre esta característica y el desempeño individual de cada ternero (OSSA et al., 2007a; OSSA et al., 2002).

Para el peso a los 18 meses (P18) solo se presentan datos de heredabilidad directa en tres estudios, con valores de $0.19,0.5$ y $0.34 \pm 0.03$ (ARBOLEDA et al., 2007; MANRIQUE, 2003; CASTAÑO et al., 2003), para las razas cruzadas (mestizos B. taurus $\mathrm{x}$ B. indicus) y Brahman puros y comerciales. Esta característica refleja la capacidad individual de los animales y es objeto de evaluación para futuros reproductores (ARBOLEDA et al., 2007). En la tabla 2 se presenta la totalidad de estudios reportados para P12, P16 y P18 meses. 
Tabla 2. Parámetros de $h_{d}{ }^{2}, h_{m}{ }^{2}$ y $h_{t}^{2}$ estimados para $\mathrm{P} 12, \mathrm{P} 16 y \mathrm{P} 18$ en características productivas en ganado tipo carne en Colombia.

\begin{tabular}{|c|c|c|c|c|c|c|c|c|c|c|}
\hline \multirow[b]{2}{*}{ AUTOR } & \multirow[b]{2}{*}{ REVISTA } & \multicolumn{3}{|c|}{ P12 } & \multicolumn{3}{|c|}{ P16 } & \multicolumn{3}{|c|}{ P18 } \\
\hline & & $\mathbf{h}_{\mathrm{d}}^{2}$ & $h_{m}{ }^{2}$ & $h_{t}^{2}$ & $\mathbf{h}_{d}{ }^{2}$ & $h_{m}{ }^{2}$ & $\mathbf{h}_{\mathrm{t}}^{2}$ & $h_{d}{ }^{2}$ & $h_{m}^{2}$ & $h_{t}{ }^{2}$ \\
\hline ARBOLEDA et al. (2007) & LRRD & & & & & & & 0,19 & & \\
\hline CASTAÑO et al.(2003) & COL CIEN PEC & & & & & & & $0,34 \pm 0,03$ & & \\
\hline MANRIQUE (2003) & COL CIEN PEC & 0,4 & & & & & & 0,5 & & \\
\hline MARTINEZ et al. (2006) & CORPOICA & & & & $0,33 \pm 0,66$ & $0,01 \pm 0,04$ & 0,38 & & & \\
\hline OSSA et al . (2007aㅡ) & CORPOICA & & & & $0,13 \pm 0,05$ & & & & & \\
\hline OSSA et al .(2002) & MVZ & & & & $0,22 \pm 0,08$ & & & & & \\
\hline
\end{tabular}

\section{Correlaciones}

El coeficiente de correlación genética es de gran importancia en el proceso de selección, pues ofrece una medida de la proporción en que los genes causan variaciones simultáneamente a dos caracteres diferentes (OSSA et al., 2007a).

Las correlaciones más frecuentes se observaron entre las características PN y PD por lo que se pueden considerar como las características productivas mas estudiadas. Los valores de correlaciones genéticas presentadas para PN y PD, oscilan entre $-0.2 \pm 0.03$ (MONTES et al., 2008a) y 0.41 (MANRIQUE, 2003), ambos para la raza Brahman. Tales variaciones y discrepancias entre valores de correlaciones para estas características dentro de la misma raza, pueden ser atribuidas a las variaciones ambientales entre lugares de ejecución de las investigaciones y en la totalidad de datos registrados para la misma; para la investigación de MONTES et al., (2008a), se utilizaron 612 registros productivos y MANRIQUE (2003) utilizo cerca de 27.600 registro en toda Colombia, sin embargo, cabe resaltar que los efectos hallador por MONTES et al., (2008a) son muy validos para su lugar de estudio (Empresa ganadera). Con relación a las correlaciones fenotípicas para PN-PD, estas registran valores de 0.14 (MONTES et al., 2008), 0.13 (MANRIQUE, 2003) y 0.16 (OSSA et al., 2007), respectivamente. Las correlaciones ambientales para el PN-PD, muestran 
registros de de 0.26 (MONTES et al., 2008) y 0.16 (OSSA et al., 2007). Respectivamente.

En la tabla 3, muestra los estudios realizados en Colombia que referencian las, las correlaciones genética, fenotípica y ambientales para características de crecimiento, en ganado tipo carne estimados en Colombia

Tabla 3. Correlaciones genéticas, fenotípicas y ambientales para características de crecimiento, en ganado tipo carne estimados en Colombia

\begin{tabular}{|c|c|c|c|c|c|c|c|c|c|}
\hline \multirow[b]{2}{*}{ AUTOR } & \multirow[b]{2}{*}{ REVISTA } & \multicolumn{8}{|c|}{ CORRELACION GENETICA } \\
\hline & & PN-PD & PN-P12 & PN-P16 & $\begin{array}{l}\text { PN- } \\
\text { P18 }\end{array}$ & $\begin{array}{l}\text { PD- } \\
\text { P12 }\end{array}$ & $\begin{array}{l}\text { PD- } \\
\text { P16 }\end{array}$ & PD-P18 & $\begin{array}{l}\text { P12- } \\
\text { P18 }\end{array}$ \\
\hline MONTES et al. (2008) & MVZ & $-0,2$ & - & - & - & - & - & - & - \\
\hline MANRIQUE (2003) & COL CIEN PEC & 0,41 & 0,16 & - & 0,13 & 0,22 & - & - & - \\
\hline CASTAÑO et al. (2003) & COL CIEN PEC & - & - & - & - & - & - & $0,55 \pm 0,17$ & - \\
\hline OSSA et al. ( 2007) & CORPOICA & 0,132 & - & 0,032 & - & - & 0,891 & - & - \\
\hline AUTOR & REVISTA & \multicolumn{8}{|c|}{ CORRELACION FENOTIPICA } \\
\hline OSSA et al. (2002) & MVZ & 0,19 & - & - & - & - & 0,045 & - & - \\
\hline MONTES et al. (2008 & MVZ & 0,14 & - & - & - & - & - & - & - \\
\hline MANRIQUE (2003) & COL CIEN PEC & 0,13 & 0,12 & - & 0,12 & 0,32 & & 0,29 & 0.56 \\
\hline CASTAÑO et al. (2003) & COL CIEN PEC & - & - & - & - & - & - & 0,37 & - \\
\hline OSSA et al. (2007) & CORPOICA & 0,161 & & 0,221 & - & - & 0,712 & - & - \\
\hline AUTOR & REVISTA & \multicolumn{8}{|c|}{ CORRELACION AMBIENTAL } \\
\hline MONTES et al. (2008) & MVZ & 0,26 & & & & & & & \\
\hline CASTAÑO et al. (2003) & COL CIEN PEC & - & & & & & & & \\
\hline OSSA et al. (2007) & CORPOICA & 0,167 & & 0.257 & & & 0.683 & 0.59 & \\
\hline
\end{tabular}

\section{Repetibilidad}

Según FALCONER (1989), la repetibilidad se define como la fracción de la varianza fenotípica que se debe a efectos genéticos y ambientales permanentes; los estimados de repetibilidad para pesos de animales en diferentes edades varían mucho, en razón de ser obtenidos en épocas diferentes, para animales de razas diferentes y criados en condiciones diversas (OSSA et al., 2008).

El $33 \%$ de los artículos evaluados estimaron valores de repetibilidad para las características $P N$ y $P D$, reportando valores de $0.05 \pm 0.02$ y $0.082 \pm 0.48$ para la raza Romosinuano (OSSA et al., 2008), $0.11 \pm 0.05$ y $0.19 \pm 0.04$ para animales de la raza Brahman (MONTES et al., 2009b) y $0.2 \pm 0.0001$ y $0.24 \pm 0.7$ para la raza CCC (MARTINEZ et al., 2006a) respectivamente. La tabla 4 presenta información detallada de los anteriores trabajos. 
Tabla 4. Parámetros de repetibilidad estimados para PN y PD en ganado tipo carne en Colombia

\begin{tabular}{|l|c|c|c|}
\hline \multicolumn{1}{|c|}{ AUTOR } & REVISTA & PN & PD \\
\hline OSSA et al. (2008) & CORPOICA & $0,05 \pm 0,02$ & $0,082 \pm 0,048$ \\
\hline MONTES et al. (2009) & COL CIEN ANIM & $0,11 \pm 0,05$ & $0,19 \pm 0,04$ \\
\hline MARTINEZ et al. $(2006)$ & CORPOICA & 0,2 & 0,24 \\
\hline
\end{tabular}

\section{Características reproductivas}

\section{Edad al primer parto}

La edad al primer parto (EPP) es una característica que refleja la eficiencia reproductiva de un hato, de igual manera las condiciones alimenticias y de manejo, y el crecimiento de una población bovina (OSSA et al., 2007b). Esta característica es de vital importancia en términos económicos para la empresa, ya cuanto más precoz fuere la edad al primer parto, mejor será la vida útil de la vaca y consecuentemente producirá mas terneros (SUAREZ et al., 2006). Para esta característica, SUAREZ et al. (2006), reportaron un valor de 0.16 $\pm 0.086\left(\mathrm{~h}_{d}{ }^{2}\right)$ y OSSA et al. (2007b) un valor de $0.16 \pm 0.08$, para la raza Romosinuano.

\section{Intervalo entre partos}

El intervalo entre partos (IEP) constituye el carácter más importante dentro de la eficiencia reproductiva de un hato, ya que incide de madera directa en el tiempo productivo de la vaca y consecuentemente en la rentabilidad del hato (OSSA et al., 2007b). Dentro de las características reproductivas, el IEP, evidencia mayor importancia, dado el interés de los investigadores en generar estimativas de heredabilidad para esta característica, pese a que esta característica siempre ha evidenciado bajos índices de herencia (VERGARA et al., 2008). 5 de los 27 estudios revisados, presentan valores de heredabilidad para IEP, siendo estos: $0.15 \pm 0.07$ (VERGARA et al., 2008), 0.0 \pm 0.06 (VERGARA, 2001), 0.096 \pm 0.08 (MONTES et al., 2009c) y 0.05 \pm 0.04 (OSSA et al., 2006) y 0.05 \pm 0.04 (OSSA et al., 2007b) para bovinos mestizos, animales de la raza Brahman (puros y comerciales) 
y raza criollas Colombianas, respectivamente. La tabla 5, presenta en conjunto los parámetros genéticos estimados para características reproductivas.

Tabla 5. Parámetros genéticos para características reproductivas en ganado tipo carne en Colombia.

\begin{tabular}{|l|c|c|c|}
\hline \multicolumn{1}{|c|}{ AUTOR } & REVISTA & IEP & EPP \\
\hline VERGARA et al. (2008) & MVZ & $\mathrm{h}_{\mathrm{d}}{ }^{2}$ & $\mathrm{~h}_{\mathrm{d}}{ }^{2}$ \\
\hline VERGARA (2001) & MVZ & $0,15 \pm 0,07$ & - \\
\hline SUARES et al. (2006) & MVZ & $0,0 \pm 0,06$ & - \\
\hline OSSA et al. (2006) & MVZ & - & $0,16 \pm 0,086$ \\
\hline MONTES et al. (2009) & LRRD & $0,05 \pm 0,04$ & - \\
\hline OSSA et al. (2007) & CORPOICA & $0,096 \pm 0,089$ & - \\
\hline OSSA et al. $(2008)$ & CORPOICA & $0,05 \pm 0,04$ & $0,16 \pm 0,086$ \\
\hline
\end{tabular}

\section{Diferencias esperadas de progenie}

Las Diferencias Esperadas de Progenie (DEP) se presentan como el valor resultante de una evaluación genética, estimado simultáneamente para cada individuo que conforma la población en control (toros, vacas y productos), Estas variables, representa la mitad del valor genético estimado del individuo y estima la capacidad de transmisión de genes de un individuo a su descendencia para una determinada característica. Además representa el diferencial en términos productivos ( $\mathrm{kg}$, Its, $\mathrm{cm}$, etc.) que se espera que la progenie de un determinado individuo produzca en promedio, con relación al promedio de la progenie de otro individuo o base genética utilizada. Las DEP son herramientas muy importantes y fáciles de ser usadas, pues son informaciones directas que expresa el valor real genético del individuo y dependen de la heredabilidad de la característica, las correlaciones con características incluidas en la evaluación, el número de datos utilizados, las relaciones entre los animales con información y la distribución de la información en el hato (BIF, 2002).

La DEP predice la diferencia que tendrán grupos de futuros hijos de animales evaluados en la misma población, esto implica que los resultados de evaluación solo pueden compararse en un mismo análisis, toros de diferentes análisis no se pueden comparar (PLASSE et al., 2004). Este tipo de evaluación ha sido poco usada en Colombia, solo 3 de los 27 artículos revisados presentas estimados de diferencias esperadas de progenie para bovinos. En la raza Brahman (CASTAÑO et al., 2003) estimando valores de cría peso a los 18 meses, en toros Brahman, encontró que estos oscilaban entre -4 y y $14.5 \mathrm{~kg}$; así mismo, Cañas et al. 
(2008), utilizando información de PD en ganado BON, calculo el promedio de DEP directas y maternas cuyos valores promedios fueron de $2.95 \pm 6.72$ y -1.02 $\pm 3.47 \mathrm{Kg}$, respectivamente; OSSA et al. (2008), utilizando información de ganado Romosinuano, calculo las DEP diferenciales entre los toros de mayor y menor potencial genético para los pesos al nacer, al destete y a los 16 meses, encontrando valores de $+4,07,+29,30 \mathrm{y}+36,04 \mathrm{~kg}$. Respectivamente. Las DEP de las vacas para los pesos al parto y al destete del ternero fueron de $+36,04 \mathrm{y}$ $+43,23 \mathrm{~kg}$. Para la edad al primer parto y el intervalo entre partos las DEP fueron de $-89,35$ y $-30,10$ días, respectivamente. Sin embargo el autor afirma, que la variabilidad genética de estos caracteres se ha mantenido, ya que los coeficientes de regresión $\left(r^{2}\right)$ tendieron a cero. La entidad que lidera esta tipo de evaluaciones en el país es ASOCEBU, generando evaluaciones genéticas con DEP para animales de la raza Brahma, cuyo objetivo es proporcionar a los criadores una herramienta para valorar genéticamente sus animales, y de esta forma poder aumentar el rendimiento de su hato a través de la utilización de toros de mayor potencial genético.(ASOCEBU, 2007).

\section{Conclusión}

Los parámetros genéticos para características de crecimiento publicados en Colombia son pocos comparados con revisiones publicadas en otros países y en temas más específicos (DE LIRA et al., 2008; RíOS et al., 2008).

Las características productivas mas evaluadas fueron el PN y PD, mientras que para las reproductivas solo se hallaron reportes para IEP y EPP.

Las razas con más investigaciones en términos genéticos son las razas criollas colombianas, sumando más del $55 \%$ de los artículos revisados, seguidos de la raza Brahman (21\%), y los bovinos mestizos (17\%).

El $33 \%$ de los artículos revisados fueron publicados en la Revista MVZ (Universidad de Córdoba), seguida por la revista Corpoica (26\%) y la Revista Colombiana de Ciencias Pecuarias (19\%); de igual manera se reportaron, pero en menor participación, publicaciones en revistas nacionales como la LRRD y la revista Colombiana de Ciencia Animal, así como también en revistas internacionales (Journal of Animal Science). 


\section{Referencias}

ASOCEBU. 2007 .Evaluación genética de toros Brahman . [citado 5 Jun 2009]. Disponible en: URL: http://asocebu.com/getdoc/9e27d008-fd7d-41aa-9163755fc66140ee/Evaluacion genetica 2007.aspx

ARBOLEDA, E.; CERON, M.; COTES, J. 2008. Heterocigosis individual y materna en poblaciones bovinas multirraciales de diferentes sistemas para producción de carne en el trópico bajo Colombiano. Revista Livestock Research for Rural Development . Vol 20(12) [acceso 4 junio 04 de 2009]. URL disponible en: http://www.Irrd.org//rrd20/12/arbl20209.htm

ARBOLEDA, E.; VERGARA, O.; RESTREPO, L. 2007. Características de crecimiento en bovinos mestizos de la costa norte Colombiana. Revista Livestock Research for Rural Development. Vol 19 (5) [acceso 14 junio 04 de 2009]. URL disponible en: http://www.Irrd.org//rrd19/5/arbo19068.htm

BIF 2002 . Guidelines For Uniform Beef Improvement Programs. Eighth Edition, The University of Georgia. EE. UU. p 161.

CAÑAS J, RAMIREZ J, ARBOLEDA O, OCHO J, VERGARA O Y CERON-MUÑOZ M 2008. Estimación de parámetros genéticos para peso al destete en ganado Blanco Oreginegro (BON) en el noroccidente Colombiano. Revista MVZ de Córdoba .Vol 13(1) [acceso 14 junio 04 de 2009]. URL disponible en: http://www.unicordoba.edu.co/revistas/revistamvz/mvz-131/V13N1A4.pdf

CASTAÑO L, GÓMEZ M Y QUIJANO J 2003. Heredabilidad y correlaciones entre peso al destete y a los dieciocho meses en un hato de ganado de carne. Revista. Colombiana de Ciencias Pecuarias Vol 16 Supl Resumen VII ENICIP[acceso 4 de agosto 2009]. URL disponible en: http://rccp.udea.edu.co/index.php/ojs/article/viewFile/142/139

DE LIRA T, ROSA M E GARNERO A 2008 .Parâmetros genéticos de características produtivas e reproductivas em zebuínos de corte (revisão). Ciência Animal Brasileira Vol 9(1) [acceso 14 de agosto 2009]. URL disponible en: http://www.revistas.ufg.br/index.php/vet/article/view/3655/3421 
DODENHOFF J, VAN VLECK L D AND GREGORY K E 1999a. Estimation of direct, maternal and grandmaternal genetic effects for weaning weigth in several breeds of beef cattle. Journal of Animal Science Volume 77 [acceso 24 de agosto 2009]. URL disponible en: http://jas.fass.org/cgi/reprint/77/4/840

DODENHOFF J, VAN VLECK L D AND WILSON E 1999B. Comparison of model to estimate genetics effects of weaning weight of Angus cattle. Journaol of Animal Science Volume 77 [acceso 28 de agosto 2009]. URL disponible en: http://jas.fass.org/cgi/reprint/77/12/3176

ELZO M, MANRIQUE C, OSSA G AND ACOSTA O 1998. Additive and nonadditive genetic variabilityfor growth traitsin the Turipana Romosinuano-Zebu multibreed herd. Journal of Animal Science Volume 76 [acceso 24 de agosto 2009]. URL disponible en: http://jas.fass.org/cgi/reprint/76/6/1539

ELZO M, MARTINEZ G, GONZALES F Y HUERTAS H 2003. Variabilidad y predicciones genéticas aditivas, no aditivas y totales para características de carne en el rebaño multirracial sanmartinero-cebú del centro de investigaciones la libertad. Revista Colombiana de Ciencias Pecuarias Vol 16 Supl Resumen VII ENICIP[acceso 24 de agosto 2009]. URL disponible en: http://rccp.udea.edu.co/index.php/ojs/article/viewFile/142/139

FALCONER D J 1980 Introducción a la genética cuantitativa. México, CECS

FALCONER D J and MACKAY T F C 1996 Introduction to Quantitative Genetics, Ed 4. Longmans Green, Harlow, Essex, UK.

FEDEGAN 2006. Y ahora que sigue para los ganaderos. En Carta Fedegán N. ${ }^{\circ}$ 112.

GALLEGO J, MARTINEZ R Y MORENO F 2006. Índice de consanguinidad y caracterización fenotípica y genética de la raza bovina Blanco Oreginegro. Revista Corpoica Vol 7(1) [acceso 24 de agosto 2009]. URL disponible en: http://www.corpoica.org.co/SitioWeb/Archivos/Revista/2 Consanguinity Coefficient BON.pdf

MADR 2008. Encuesta Nacional Agropecuaria [acceso 25 de agosto 2009]. URL disponible en:

http://www.agronet.gov.co/www/docs agronet/20095694411 ENA 2008.pdf 
MANRIQUE C 2003. Parámetros genéticos de la raza Brahman en Colombia. Revista Colombiana de Ciencias Pecuarias Vol 16 Supl Resumen VII ENICIP[acceso 24 de agosto 2009]. URL disponible en: http://rccp.udea.edu.co/index.php/ojs/article/viewFile/142/139

MARTINEZ R Y PEREZ J 2006B. Parámetros y tendencias genéticas para características de crecimiento en ganado criollo Romosinuano. Revista Corpoica 7(1) [acceso 24 de agosto 2009]. URL disponible en: http://www.corpoica.org.co/SitioWeb/Archivos/Revista/3 Genetic Parameters Ro $\underline{\text { mosinuano.pdf }}$

MARTINEZ R, PEREZ J Y HERAZO T 2006ª. Evaluación fenotípica y genética para carateristicas de crecimiento en la raza criolla Colombiana Costeño con Cuernos. Revista. Corpoica Vol 7(2) [acceso 24 de agosto 2009]. URL disponible en:

http://www.corpoica.org.co/SitioWeb/Archivos/Revista/2 EvalFenotipGrowthCCC RevCorpoica v7N2 2006.pdf

MONTES D, VERGARA O Y PRIETO E 2009 a. Una nota sobre los factores que afectan el peso al destete en ganado cebú Brahman. Revista Livestock Research for Rural Development. Vol 21(9) [acceso 17 octubre de 2009]. URL disponible en: http://www.Irrd.org//rrd21/9/mont21141.htm

MONTES D, VERGARA O Y PRIETO E 2009 b. Una nota sobre La estimación Del intervalo entre partos en hembras bovinas de ganado Brahman. Revista Livestock Research for Rural Development. Vol 21(1) [acceso 17 octubre de 2009]. URL disponible en: http://www.Irrd.org//rrd21/1/mont21002.htm

MONTES D, VERGARA O, PRIETO E Y BARRAGÁN W 2009. Estimación de La repetibilidad y factores que afectan el peso al nacer y al destete en ganado bovino cebú Brahman. Revista Colombiana de Ciencia Animal Vol 1(1) [acceso 17 octubre de 2009]. URL disponible en: http://www.recia.edu.co/documentosrecia/MONTES DONICER2009.pdf

MONTES D, VERGARA O, PRIETO E Y RODRÍGUEZ A 2008. Estimación de los parámetros genéticos para peso $A L$ nacer y al destete en ganado bovino de la 
raza brahmán. Revista $M V Z$ de Córdoba Vol 13(1) [acceso 17 octubre de 2009]. URL disponible en: http://www.unicordoba.edu.co/revistas/revistamvz/mvz131/N13N1A8.pdf

OSSA G Y PEREZ J 2002. Efecto del medio y de la herencia sobre los pesos al nacer, destete y 16 meses de edad en la raza costeño con cuernos. Revista MVZ de Córdoba Vol 7(1) [acceso 17 octubre de 2009]. URL disponible en: http://www.unicordoba.edu.co/revistas/revistamvz/MVZ-71/143.pdf

OSSA G, SUAREZ M Y PEREZ 2008 Valores genéticos de caracteres productivos y reproductivos en bovinos Romosinuanos. Revista Corpoica Vol 9(1) [acceso 17 octubre de 2009]. URL disponible en: http://www.corpoica.org.co/SitioWeb/Archivos/Revista/12.Valoresgenticosdecaract eresproductivosyreproductivosenbovinosRomosinuano.pdf

OSSA G, SUAREZ M Y PEREZ J 2005a . Efectos del medio y la herencia sobre el peso al nacimiento en terneros de la raza romosinuano. Revista MVZ de Córdoba Vol 10(1) [acceso 17 octubre de 2009]. URL disponible en http://www.unicordoba.edu.co/revistas/revistamvz/mvz-101/101-6.pdf

OSSA G, SUAREZ M Y PEREZ J 2005B Efecto del medio y la herencia sobre el peso al destete en terneros de la raza romosinuano. Revista MVZ de Córdoba Vol 10(2) [acceso 17 octubre de 2009]. URL disponible en: http://www.unicordoba.edu.co/revistas/revistamvz/mvz-102/102-9.pdf

OSSA G, SUAREZ M Y PEREZ J 2006 Factores ambientales y genéticos relacionados con el intervalo entre partos en la raza romosinuano. Revista MVZ de Córdoba Vol 11(2) [acceso 17 octubre de 2009]. URL disponible en: http://www.unicordoba.edu.co/revistas/revistamvz/mvz-112/112-4.pdf

OSSA G, SUAREZ M Y PEREZ J 2007a Efecto del medio y la herencia sobre el peso al naciminento, destete y a los 16 meses de edad en terneros de la raza Romosinuano. Revista Corpoica 8(2) [acceso 17 octubre de 2009]. URL disponible en:

http://www.corpoica.org.co/SitioWeb/Archivos/Revista/11.Efectosdelmedioylaheren cia.pdf 
OSSA G, SUAREZ M Y PEREZ J 2007b Factores ambientales y genéticos que influyen la edad al primer parto y el intervalo entre partos en hembras de la raza criolla Romosinuano. . Revista Corpoica 8(2) [acceso 17 octubre de 2009]. URL disponible en:

http://www.corpoica.org.co/SitioWeb/Archivos/Revista/10.Factoresambientalesyge $\underline{\text { nticos.pdf }}$

PLASSE D, ARANGO J, FOSSI H, CAMARIPANO L, LLAMOZAS G, PIERRE A, Y ROMERO R 2004 Genetic and non-genetic trends for calf weights in a Bos indicus herd upgraded to pedigree Brahman. Revista Livestock Research for Rural Development . Vol 16(7) [acceso 17 octubre de 2009]. URL disponible en: http://www.Irrd.org//rrd16/7/plas16046.htm

QUINTERO J, TRIANA J, QUIJANO J Y ARBOLEDA E 2007 Influencia de la inclusión del efecto materno en la estimación de parámetros genéticos del peso al destete en un hato de ganado de carne. Revista Colombiana de Ciencias Pecuarias Vol 20 [acceso 17 octubre de 2009]. URL disponible en: http://rccp.udea.edu.co/index.php/ojs/article/viewFile/268/265

RIOS A 2008 Estimadores de parâmetros genéticos para características de crecimineto predestete en bovinos (revisión). Revista Técnica Pecuaria de México Vol 46(1) [acceso 17 octubre de 2009]. URL disponible en: http://www.tecnicapecuaria.org.mx/trabajos/200801145077.pdf

SUAREZ M, OSSA G Y PEREZ J 2006 Factores ambientales y genéticos que influyen sobre la edad al primer parto en hembras de la raza romosinuano. Revista MVZ de Córdoba Vol 11(1) [acceso 17 octubre de 2009]. URL disponible en:

http://www.unicordoba.edu.co/revistas/revistamvz/mvz-111/111-7.pdf

VERGARA O 2001 Estimación de la heredabilidad del intervalo entre partos en ganado cebu. Revista MVZ de Córdoba Vol 6(1) [acceso 17 octubre de 2009]. URL disponible en: http://www.unicordoba.edu.co/revistas/revistamvz/MVZ61/48.pdf 
VERGARA O, CERON-MUÑOZ M, ARBOLEDA E, OROZCO Y OSSA G 2009 Direc genetic, maternal genetic, and heterozygocity effec on weaning weigth in a Colombian multibreed beef cattle population. Journal of Animal Science Volume 87 p 516-521. Disponible en: http://jas.fass.org/cgi/content/abstract/jas.2007-0636v1

VERGARA O, CERON-MUÑOZ M, HURTADO N, ARBOLEDA E, GRANADA J Y RUA C 2008 Estimación de La heredabilidad del intervalo entre partos en ganado cruzado. Revista MVZ de Córdoba Vol 13(1) [acceso 17 octubre de 2009]. URL disponible en: http://www.unicordoba.edu.co/revistas/revistamvz/mvz131/V13N1A9.pdf 